PALAVRAS. Revista de Epistemología, Metodología y Ética del Psicoanálisis ISSN: 2468-9831

Licencia de Cultura Libre Creative Commons (C-BY 4.0).

https://revistas.unlp.edu.ar/palavras

palavras@outlook.com.ar

\title{
LA CIENCIA DEL PSICOANALISIS Y EL PSICOANALISIS DE LA CIENCIA
}

\section{https://doi.org/10.24215/24689831e035}

\section{Pablo Cazau}

\begin{abstract}
Within the broad spectrum of topics that could relate psychoanalysis to scientific knowledge, this article addresses two fundamental que stions: 1) the conditions under which psychoanalysis can be considered as science, and 2) psychoanalytic explanations about scientific knowledge and, in particular, those offered by Sigmund Freud himself and later, by Gastón Bachelard, who will investigate the roots of scientific discourse in the psychodynamics of the unconscious.

Psychoanalysis "as science" and psychoanalysis "of science" are thus two alternative and in a sense complementary approaches where both belief systems scrutinize each other: science looking at psychoanalysis, and psychoanalysis looking at science. It is concluded that at heart it is not a matter of opposed approaches but rather of two approaches oriented towards the same objective, which is the construction of an epistemology of psychoanalysis.
\end{abstract}

Key words: epistemology - science - psychoanalysis.

\section{Resumen}

Dentro del amplio espectro de temas que podrian relacionar el psicoanálisis con el conocimiento científico, en este artículo se rescatan dos cuestiones fundamentales: 1) las condiciones bajo las cuales el psicoanálisis puede ser considerado comociencia,y2) las explicaciones psicoanalíticas acerca del conocimiento científico y, en particular, las ofrecidas por el mismo Sigmund Freudy más tarde, por Gastón Bachelard, que indagarán las raíces del discurso científico en la psicodinámica del inconciente.

El psicoanálisis "como ciencia" y el psicoanálisis "de la ciencia" son así dos enfoques alternativos y en cierto sentido complementarios donde ambos sistemas de creencias se escrutan mutuamente: la ciencia mirando al psicoanálisis, y el psicoanálisis mirandoa la ciencia. Se concluye que en el fondo no se trata de enfoques contrapuestos sino más bien de dos planteos orientados hacia el mismo objetivo que es la construcción de una epistemología del psicoanálisis.

Palabras clave: epistemología - ciencia psicoanálisis.

\section{Cómo citar este artículo:}

Cazau, P. (2020). La ciencia del psicoanálisis y el psicoanálisis de la ciencia. Palauras.

Revista de Epistemología, Metodología y Ética del Psicoanálisis, (6), e035. https://doi.org/10.24215/24689831e035 


\section{LA CIENCIA DEL PSICOANÁLISIS Y EL PSICOANÁLISIS DE LA CIENCIA}

Pablo Cazau ${ }^{1 *}$

\section{La concepción freudiana de ciencia}

Por lo general, los epistemólogos más reconocidos se han formado en las llamadas ciencias duras. Así Kuhn, Lakatos, Bachelard y Piaget se formaron respectivamente en la física, la matemática, la química y la biología. Si bien Sigmund Freud no tuvo una trayectoria como epistemólogo, sus pronunciamientos respecto del conocimiento científico sufrieron la influencia en parte de las ciencias duras -especialmente la fisica y la biología-, y en parte de las llamadas ciencias blandas que, como la psicología, ya desde el siglo XIX comenzaban a reclamar su estatus científico.

Freud fue un empecinado defensor de la cosmovisión científica de su época, entendiendo cosmovisión como "una construcción intelectual que soluciona de manera unitaria todos los problemas de nuestra existencia a partir de una hipótesis suprema" (Freud, 1933/1995, p. 146). Inspirándose en la doctrina positivista de Comte, Freud menciona tres fases en la historia de las cosmovisiones: la animista, la religiosa y la científica, indicando que puede seguirse a través de ellas la evolución de la 'omnipotencia de las ideas' que había observado en los obsesivos. Dice así que "en la fase animista se atribuye el hombre a sí mismo la omnipotencia; en la etapa religiosa la cede a los dioses, sin renunciar de todos modos muy seriamente a ella, pues se reserva el poder de influir sobre los dioses de manera de hacerlos actuar

\footnotetext{
1* Universidad Favaloro. Carrera de Ortodoncia y Ortopedia Maxilar. Comisión de Ase soría Científica. Argentina. Mail: pcazau@gmail.com
} 
conforme a sus deseos. En la concepción científica del mundo no existe ya lugar para la omnipotencia del hombre, el cual ha reconocido su pequeñez y se ha resignado a la muerte y sometido a todas las demás necesidades naturales" (Freud, 1913/1968, p. 558).

Freud caracteriza la cosmovisión científica a partir de cinco rasgos, de los cuales los tres primeros dejan traslucir la impronta del positivismo más estricto de su época.

a) La ciencia aspira a ser un saber racional.- Señala Freud que "nuestra mejor esperanza para el futuro es que el intelecto -el espíritu científico, la razón- establezca con el tiempo la dictadura dentro de la vida anímica. La esencia de la razón garantiza que en tal caso no dejaría de asignar su lugar debido a las mociones afectivas de los seres humanos y a todo lo comandado por ellas" (Freud, 1933/1995, p. 158).

b) La ciencia es un saber empírico y verificable.- La exigencia de verificabilidad es introducida Freud cuando señala que el pensamiento científico "somete a riguroso examen la certeza de las percepciones sensoriales sobre las que edifica sus inferencias, se procura nuevas percepciones inalcanzables con los medios cotidianos $\mathrm{y}$, variando deliberadamente ciertos experimentos, aísla las condiciones de esas experiencias nuevas" (Freud, 1933/1995, p. 157).

c) La ciencia es un saber neutral.- La neutralidad del saber científico apunta básicamente a la necesidad de que el investigador mantenga su objetividad y no se deje influenciar por prejuicios o valoraciones afectivas. "El pensar científico no es diverso por su esencia de la actividad normal del pensamiento que todos nosotros, creyentes y no creyentes, aplicamos en nuestros menesteres vitales. Sólo en algunos rasgos ha cobrado particular relieve: (...) se empeña por mantener cuidadosamente alejados los factores individuales y las influencias afectivas" (Freud, 1933/1995, p. 157). 
d) La ciencia es un saber cambiante.- Sostiene Freud que "por su carácter eternamente incompleto e insuficiente, la ciencia está condenada a confiar para su salud en nuevos descubrimientos y concepciones [aun cuando] sabemos bien que en la historia de la investigación científica las innovaciones tropezaron a menudo con una intensa y obstinada resistencia que luego se demostró injusta, porque la novedad era valiosa y sustantiva" (Freud, 1923/1995, p. 227).

e) La ciencia es un saber impreciso y perfectible.- Para Freud, la ciencia "carece de los caracteres de precisión, inmutabilidad e infalibilidad, tan ansiados por el pensamiento humano" (Freud, 1926/1995, p. 179). El creador del psicoanálisis describe con mayor detalle esta tarea del científico cuando señala que "muchas veces hemos oído sostener el reclamo de que una ciencia debe construirse sobre conceptos básicos claros y definidos con precisión. En realidad, ninguna, ni aún la más exacta, empieza con tales definiciones" (Freud, 1915/1995, p. 113). Agrega que el comienzo correcto de la actividad científica consiste más bien en describir fenómenos que luego son agrupados, ordenados e insertados en conexiones. Ya para la descripción misma es inevitable aplicar al material citas ideas abstractas que se recogieron de alguna otra parte, no de la sola experiencia nueva.

\section{El psicoanálisis como ciencia}

Al ubicar Freud (1938/1968) el psicoanálisis dentro de la cosmovisión científica por considerarla la rama de una ciencia como la psicología, le adscribirá también ciertos rasgos del saber científico en general mencionados ut supra: racional, empírico, verificable, neutral, cambiante, incierto y perfectible.

a) El psicoanálisis como saber racional.- Cuando Freud especula con que algún día el hombre podrá regirse por la razón, ubica al psicoanálisis como otro esfuerzo más encaminado hacia ese objetivo. Freud sugiere aqui el pasaje de un sujeto pulsional a un sujeto racional a través de un proceso sublimatorio, aunque preservándolo como entidad deseante. El 
psicoanálisis se presenta a sí mismo como la tercera herida narcisista de la humanidad (luego de Copérnico y Darwin) al mostrar descarnadamente la irracionalidad humana, pero al mismo tiempo como la posibilidad de curar la herida y acceder el pensamiento racional.

Por otra parte, es habitual adscribirle a la razón un pensamiento determinista según el cual todo en la realidad está determinado y no hay lugar para el azar. En su conferencia sobre actos fallidos (1915-1917/1995) Freud explica que no basta hablar simplemente de determinismo, sino concretamente de determinismo psíquico, ya que admite ciertas creencias y prejuicios donde las personas recurren a un determinismo religioso de origen divino, o a un determinismo de carácter simplemente fisiológico, como cuando una equivocación queda explicada simplemente por un estado de fatiga, de sobreexcitación o desatención. En su conferencia 6 sobre condiciones y técnicas de la interpretación, Freud es contundente al respecto: "semejante creencia es por completo anticientífica y debe desaparecer ante la reivindicación de un determinismo psíquico" (1915$1917 / 1995)$.

b) El psicoanálisis como saber empírico y verificable.- Aparece en Freud manifiestamente esta idea en afirmaciones tales como "el psicoanálisis basa sus afirmaciones en un cierto número de hechos" (Freud, 1938/1968, p. 443); y "el psicoanálisis ha construido, sobre la base de una gran cantidad de observaciones e impresiones, algo como una teoría" (Freud, 1917/1968, p. 1108).

Debe tenerse en cuenta que, como teoría que es, el psicoanálisis es un entramado de proposiciones vinculadas lógicamente desde los enunciados más generales (los puntos de vista económico, dinámico y tópico de la metapsicologia) hasta los más particulares (referidos a hechos), y pasando por una serie de otros enunciados de nivel teórico intermedio (por ejemplo los relacionados con las pulsiones o la libido) (Cazau, 2013). 
A propósito de la introducción de conceptos presuntamente especulativos como libido yoica y libido objetal, Freud señala que la ciencia no debe ser una teoría especulativa sino una disciplina construida sobre la interpretación de los hechos, justificando aquellos conceptos teóricos sobre esta base. El fundamento de la ciencia no son las especulaciones sino la observación (Freud, 1914/1995, p. 75).

La exigencia de la verificabilidad se apoya sobre la idea de verdad. Freud sostiene que el afán de la ciencia "es lograr la concordancia con la realidad (...) y llamamos 'verdad' a esta concordancia con el mundo exterior objetivo" (Freud, 1933/1995, p. 157). Sin embargo, reconoce la importancia de un mundo interior subjetivo, lo que se evidencia en ciertas afirmaciones sobre las fantasias infantiles y sobre la resignificación terapéutica.

Así, en la Conferencia 23 (Freud, 1916/1995) sostiene que las experiencias infantiles fantaseadas pueden ser tan patógenas como las experiencias infantiles realmente acontecidas en el mundo objetivo. Asimismo, en el pensamiento freudiano es posible identificar lo que podría llamarse una verdad terapéutica en la medida en que considera que la cura se alcanza cuando el paciente ha podido reconstruir 'una' historia de su pasado, que no tiene necesariamente que ser 'la' historia real y objetiva, lo realmente acontecido (Cazau, 2016).

El psicoanálisis como saber empírico y verificable puede apreciarse también en la influencia que ejerció Ernest Mach (1838-1916) sobre el pensamiento freudiano, destacada por Assoun.

En el primer párrafo de "Pulsiones y destinos de pulsión" (1915/1995), Freud enfatiza que "el verdadero principio de la actividad científica consiste... en la descripción de fenómenos, que luego son agrupados, ordenados y relacionados entre sí" (Freud, 1915/1995).

Assoun (2001) encuentra aquí llamativas coincidencias, incluso literales, con un texto de Mach (1905/1948, p. 172) cuando éste hace referencia a la 
tarea de agrupar, ordenar y relacionar los fenómenos. Tal vez la única diferencia es que Mach especifica un método adecuado para tales propósitos y que está vinculado con los esquemas experimentales de Francis Bacon y John Stuart Mill, no mencionados explícitamente.

Freud menciona a Mach en varias otras ocasiones (por ejemplo en una carta a Fliess del 12-6-1900 y en "Mi relación con Josef Popper-Lynkeus" de 1932) (Freud, 1900/1968, 1932/1995). En ambos casos Freud destaca su coincidencia con el físico austríaco cuando aborda el problema de la deformación onírica tomando como referencia su obra "Analyse der Empfindungen" (Mach, 1886/1987).

c) El psicoanálisis como saber neutral.- Para Freud "el psicoanálisis es un método de investigación, un instrumento neutral como lo es, por ejemplo, el cálculo infinitesimal. Si con ayuda de este último un físico llegara a la conclusión de que transcurrido cierto lapso la Tierra desaparecerá, es evidente que se vacilará en atribuir al cálculo las mismas tendencias destructivas y en proscribirlo por ellas" (Freud, 1927 / 1995, p. 36);

Freud se ha referido también a la neutralidad en el análisis, entendiendo por tal cierta actitud que el analista debe mantener durante la cura, como por ejemplo no dejarse influenciar por prejuicios religiosos o morales, o bien no prejuzgar acerca de la importancia de ciertos tramos del discurso del paciente: los hechos no deberian amoldarse a la teoría sino a la inversa. Esta neutralidad analítica es un requisito de la cura, pero también un requisito del psicoanálisis como teoría.

d) El psicoanálisis como saber cambiante.- Para Freud el progreso del conocimiento no tolera rigidez alguna, y tampoco en las definiciones. Como lo enseña palmariamente el ejemplo de la física, también los 'conceptos básicos' fijados en definiciones experimentan un constante cambio de contenido" (Freud, 1915/1995, p. 113). Así, por ejemplo, la representación espacial del aparato psíquico es una representación auxiliar como hay 
tantas en las ciencias, que Freud llega a calificar incluso de una ficción sujeta permanentemente a revisiones (Freud, 1926/1995, p. 182).

A lo largo del pensamiento freudiano puede apreciarse este carácter cambiante cuando Freud, exigido por los hechos clínicos, se ve obligado a pasar de una primera tópica a una segunda tópica, de una primera teoría pulsional a una segunda teoría pulsional o de una primera teoría de la angustia a una segunda teoría de la angustia (Cazau, 2018).

e) El psicoanálisis como saber impreciso y perfectible.- Para Freud el psicoanálisis como teoría va construyéndose no sólo a partir de los hechos de la clínica sino también a partir de ideas vagas, imprecisas como por ejemplo la idea de la represión primaria. Sin embargo, advierte: "el psicoanálisis no es hijo de la especulación sino el resultado de la experiencia; y por esa razón, como todo nuevo producto de la ciencia, está inconcluso" (Freud, 1913/1995, p. 211).

Finalmente, puede ser necesario consignar que Freud no entró en la confrontación ciencias naturales versus ciencias sociales que comenzó a tomar vuelo hacia fines del siglo XIX, y más específicamente en la confrontación explicación versus interpretación, limitándose a proponer la segunda como una variante de la primera. En efecto, como indica Assoun "esto es precisamente lo que una epistemología freudiana debe tomar en cuenta: el hecho de que la emergencia de un punto de vista interpretativo no haya tenido ningún efecto polémico en la tesis freudiana de la primacía de la explicación. En ningún momento la interpretación, por más grande que sea su importancia clínica, implicó en la concepción que Freud forja de su propia episteme una rectificación en su sentido hermeneutista. Lejos de transferir el psicoanálisis al campo de las ciencias hermenéuticas, la Traumdeutung [Interpretación de los sueños] no modifica en absoluto, en apariencia, la identidad de la Naturwissenschaft [ciencias naturales]. Esta sigue basada en el cómo su procedimiento principal y por así decirlo regio. Esto implica, pues, que la Deutung [interpretación] freudiana se representa 
efectivamente en Freud como no disruptiva con el erklären [explicación] incluso que la interpretación se plantea como una variante de la explicación” (Assoun, 2001, p. 44).

\section{E1 psicoanálisis de la ciencia}

Freud 'psicoanalizó' a la ciencia cuando sostuvo que los procesos psicodinámicos de origen pulsional pueden desembocar, vía sublimación, en una producción científica. La sublimación es uno de los destinos de las pulsiones (Freud, 1915/1995), y se refiere a un proceso por el cual ciertas actividades culturales como la ciencia, sin aparente relación con la sexualidad, se constituyen en una vía de descarga de la energía pulsional sexual hacia un nuevo fin.

La psicología del científico se constituye así en otro capítulo importante de la epistemología a pesar de las denuncias de psicologismo de Popper cuando enfatiza que "la cuestión acerca de cómo se le ocurre una idea nueva a una persona -ya sea un tema musical, un conflicto dramático o una teoría científica-, puede ser de gran interés para la psicología empírica, pero carece de importancia para el análisis lógico del conocimiento científico" (Popper, 1967, p. 30). Otros autores rescatan, en cambio, el valor del análisis psicoanalítico del discurso científico para la epistemología, como por ejemplo Gastón Bachelard (1884-1962), cuando se refiere a un 'psicoanálisis del conocimiento objetivo' (Bachelard, 1972) y donde propone que el científico es, además de una máquina de pensar, un ser humano.

Para el filósofo francés existen ciertos factores que dificultan el desarrollo de la ciencia directamente relacionados con la subjetividad del científico, y que Bachelard llamará obstáculos epistemológicos. Se trata de creencias, habitualmente inconcientes, que requieren ser concientizadas y superadas para que el conocimiento científico avance hacia etapas de mayor objetividad. Bachelard acomete entonces la tarea de 'psicoanalizar' la ciencia para poner al descubierto aquellos obstáculos. 
Desde ya, Bachelard no propuso que los científicos vayan al psicoanalista a hacerse una especie de control epistemológico. El filósofo francés encaró en cambio la tarea de analizar psicoanalíticamente los textos científicos, especialmente los de la Edad Medieval y Moderna, y centrándose sobre todo en temas de fisica, química y biología, aunque también es posible identificarlos en otras disciplinas.

Los obstáculos epistemológicos son confusiones, entorpecimientos, prejuicios que se dan en el acto mismo de conocer, generando una inercia que tiende a perpetuar lo ya conocido, y cerrando las puertas al nuevo conocimiento. Ello ocurre porque las creencias que operan como obstáculos epistemológicos están altamente valorizadas, los que las torna dificiles de erradicar.

Bachelard (1972) identificó, entre otros, dos obstáculos epistemológicos fundamentales ubicados en extremos opuestos: la experiencia básica y el conocimiento general. La experiencia básica consiste en aferrarse a lo singular, pintoresco, fútil o anecdótico de los fenómenos, sin captar lo esencial y sin ejercer una crítica sobre los datos brutos de los sentidos. La raíz de esta actitud debe buscarse en la tendencia humana hacia el espectáculo de los fenómenos interesantes o vistosos que permitan canalizar deseos y pasiones. En el otro extremo, el conocimiento general consiste en hacer generalizaciones apresuradas. Como se ve los obstáculos se presentan de a pares y además hay una tendencia a querer superar uno de los extremos intentando alcanzar el otro.

Bachelard explicó la raíz de los obstáculos epistemológicos desde el psicoanálisis cuando los vinculó con procesos pulsionales inconcientes, pero también lo hizo desde lo que luego se llamaría la psicología cognitiva al describirlos en términos de distorsiones del pensamiento. Cabe recordarque Bachelard expuso sus ideas en 1938, cuando aún no existia la psicología ni la psicoterapia cognitiva tal como hoy es conocida. 
Es posible trazar sucintamente una comparación entre Mach y Bachelard. Por ejemplo, la experiencia básica de Bachelard se corresponde con las ilusiones de Mach, y ambos coinciden en proponerlas como obstáculos para el desarrollo del conocimiento científico. En un capítulo sugestivamente titulado "La exuberancia de las ideas", el físico austríaco señala que "...las asociaciones de ideas, sobre las cuales descansa la adaptación del pensamiento a los hechos, dependen del azar... Si circunstancias favorables han dirigido las ideas de tal manera que su curso sigue o se adelanta a los hechos, concluiremos en la ciencia. Pero las circunstancias desfavorables pueden dirigir la atención sobre cosas que no son esenciales y llevar a asociaciones de ideas que no corresponden a los hechos; entonces somos conducidos al error" (Mach, 1905/1948, p. 85).

Ernest Mach detalla más la cuestión, indicando que "nuestras percepciones físicas nos exponen a numerosos errores o ilusiones. Un bastón sumergido oblicuamente en el agua se lo v quebrado y un observador que no estuviese al corriente, podría creer que al tocar ese bastón parecería quebrado... Todas las ilusiones de este género son debidas a que ignoramos las circunstancias en las que una percepción se produce, y ... nuestra imaginación completa lo que nos proporciona la experiencia de la manera que nos es más habitual... Así, lo que conduce a oponer la apariencia a la realidad, el fenómeno y la cosa, es la confusión de las percepciones producidas por las circunstancias más diferentes con las percepciones producidas por circunstancias perfectamente determinadas" (Mach, $1905 / 1948$, p. 23).

\section{Conclusiones}

Un examen preliminar de las dos miradas propuestas revela que en el fondo no se trata de enfoques alternativos o contrapuestos sino más bien de dos enfoques orientados hacia el mismo objetivo que es la construcción de una epistemología del psicoanálisis, vale decir, hacia la consideración de la teoría psicoanalítica como producción científica. El Freud epistemólogo, por 
caso, no sólo se ha ocupado de describir el saber científico vigente en su época, sino que también se preocupó por caracterizar al psicoanálisis como un saber moldeado por la cosmovisión positivista en el sentido amplio que incluye también a Mach (el psicoanálisis como ciencia) y a la vez como un saber determinado por la dinámica del inconciente (el psicoanálisis de la ciencia).

Por extensión, ese producto final llamado ciencia está entonces básicamente determinado por factores universales que tienen que ver con el modo de funcionamiento de la mente, pero también por factores coyunturales que tienen que ver con la peculiar cosmovisión sobre la ciencia surgida en una determinada época histórica.

\section{Referencias}

Assoun, P. (2001). Introducción a la epistemología freudiana (6 edición). Siglo XXI editores.

Bachelard, G. (1972). La formación del espiritu científico. Contribuciones a un psicoanálisis del conocimiento objetivo $\left(2^{\circ}\right.$ edición). Siglo XXI.

Cazau, P. (2013). La lógica del discurso científico. Biblioteca Redpsicología.

Cazau, P. (2016). Consideración filosófica sobre la realidad y el conocimiento. Biblioteca Redpsicología.

Cazau, P. (2018). Freud y Lacan desde cero. Edición del autor.

Freud, S. (1900/1968). Carta a Fliess del 12-6-1900. En Obras Completas. Biblioteca Nueva. 
Freud, S. (1913/1968). Tótem y tabú. En Obras Completas. Biblioteca Nueva.

Freud, S. (1913/1995). Sobre psicoanálisis. En Obras Completas. Amorrortu.

Freud, S. (1914/1995). Introducción al narcisismo. En Obras Completas. Amorrortu.

Freud, S. (1915/1995). Pulsiones y destinos de pulsión. En Obras Completas. Amorrortu.

Freud, S. (1915-1917/1995). Lecciones introductorias al psicoanálisis: Conferencia 2: Los actos fallidos. En Obras Completas. Amorrortu.

Freud, S. (1916/1995). Conferencia 23: Los caminos de la formación del síntoma. En Obras Completas. Amorrortu.

Freud, S. (1917/1968). Una dificultad del psicoanálisis. En Obras Completas. Biblioteca Nueva.

Freud, S. (1923/1995). Josef Popper-Lynkeus y la teoría del sueño. En Obras Completas. Amorrortu.

Freud, S. (1926/1995). ¿Pueden los legos ejercer el análisis? En Obras Completas. Amorrortu.1995.

Freud, S. (1927/1995). El porvenir de una ilusión. En Obras Completas. Amorrortu.

Freud, S. (1932/1995). Mi relación con Josef Popper-Lynkeus. En Obras Completas. Amorrortu. 
Freud, S. (1933/1995). 35 Conferencia: En torno a una cosmovisión. En Obras Completas. Amorrortu.

Freud, S. (1938/1968). Algunas lecciones elementales de psicoanálisis. En Obras Completas. Biblioteca Nueva.

Mach, E. (1905/1948). Conocimiento y error. Espasa-Calpe.

Mach, E. (1886/1987). Die Analyse der Empfindungen und das Verhältnis des Physischen zum Psychischen, Jena. En El análisis de las sensaciones. Alta Fulla Editorial.

Popper, K. (1967). Conjeturas y refutaciones. El desarrollo del conocimiento científico. Paidós.

Recibido: 21/04/2020

Revisado: $16 / 07 / 2020$

Aprobado: 08/08/2020 